\title{
Modulated Scale-free Network in the Euclidean Space
}

\author{
S. S. Manna ${ }^{1}$ and Parongama Sen ${ }^{2}$ \\ ${ }^{1}$ Satyendra Nath Bose National Centre for Basic Sciences Block-JD, Sector-III, Salt Lake, Kolkata-700098, India \\ ${ }^{2}$ Department of Physics, University of Calcutta, 92 Acharya Prafulla Chandra Road, Kolkata 700009, India.
}

\begin{abstract}
A random network is grown by introducing at unit rate randomly selected nodes on the Euclidean space. A node is randomly connected to its $i$-th predecessor of degree $k_{i}$ with a directed link of length $\ell$ using a probability proportional to $k_{i} \ell^{\alpha}$. Our numerical study indicates that the network is Scale-free for all values of $\alpha>\alpha_{c}$ and the degree distribution decays stretched exponentially for the other values of $\alpha$. The link length distribution follows a power law: $D(\ell) \sim \ell^{\delta}$ where $\delta$ is calculated exactly for the whole range of values of $\alpha$.
\end{abstract}

PACS numbers: 05.10.-a, 05.40.-a, 05.50.+q, 87.18.Sn

Statistical properties of many different networks are being studied recently with much interests. Examples include the World-wide web (WWW) [1], the internet structure [2], neural networks [3], collaboration network [1] etc. Broadly these networks are classified into four different groups, namely, (i) Networks on regular lattices (ii) Random networks [5] (iii) Small-world networks (SWN) [6] and (iv) Scale-free networks (SFN) [7, 8].

It has been observed that the degree distributions of nodes for two very important networks e.g., World-wide web [1] which is a network of webpages (nodes) and the hyperlinks (links) among various pages and the internet network [2] of routers or autonomous systems follow power law as:

$$
P(k) \sim k^{-\gamma} .
$$

These networks are called Scale-free networks and the exponent $\gamma$ varies between 2 and 3 for these networks. Barabási and Albert (BA) proposed a simple model for an evolving SFN that has the following two essential ingredients, namely: (i) A network grows from an initial set of $m_{o}$ nodes with $m<m_{o}$ links among them. Further, at every time step a new node is introduced and is randomly connected to $m$ previous nodes. (ii) Any of these $m$ links of the new node introduced at time $t$ connects a previous node $i$ with an attachment probability $\pi_{i}(t)$ which is linearly proportional to the degree $k_{i}(t)$ of the $i$-th node at time $t$

$$
\pi_{i}^{B A}(t) \sim k_{i}(t) .
$$

For BA model $\gamma=3$ [8].

The physical distance, or the Euclidean distance between the nodes plays an important role in cases like electrical networks, internet or even in postal and transports networks etc. In these networks one tries to minimize the lengths of the connections e.g., electrical wires, ethernet cables or say travel distances of postal carriers. Static networks, in which connection probabilities depend on the Euclidean distance have already been considered in the context of small-world properties [9]. Recently a number of interesting works in the context of SFN have been published 10, 11.
Study of internet's topological structure is important for designing efficient routing protocols and modelling internet traffic. Waxman model describes internet with exponentially decaying link length distribution: $D(\ell) \sim$ $\exp \left(-\ell / \ell_{0}\right)$ [12]. Faloutsos et. al. observed the scale-free degree distribution of the internet [2]. Yook et. al. observed nodes of the router level network maps of North America are distributed on a fractal set and the link length distribution is inversely proportional to the link lengths 13]. They also argued that a competition exists beteen the preferential attachment of the nodes and the weightage of the link lengths 13 .

In this paper our aim is to study how a scale-free network defined on the Euclidean space behaves when the usual BA attachment probability as in eqn. (2) is modulated by a link length $\ell$ dependent factor $\ell^{\alpha}$. Our important observation is even for the uniform random distribution of nodes we obtain the power law variation of the link length distribution for all values of $\alpha$ including the empirically observed inverse variation where as the Waxman's exponential behaviour is never observed. We argue that for a country with homogeneously distributed router density, our results seem to be important.

Specifically in two-dimensions, we consider an unit square area on the $x-y$ plane. Randomly selected points within this area are the nodes of the network. The network grows by systematically introducing one node at a time with randomly chosen coordinates $(x, y) ; 0 \leq x, y<$ 1 with uniform probabilities. The attachment probability that the new node introduced at time $t$ would be connected to its $i$-th predecessor $(0 \leq i \leq t-1)$ is:

$$
\pi_{i}(t) \sim k_{i}(t) \ell^{\alpha}
$$

where, $\ell$ is the Euclidean distance between the $t$-th and the $i$-th node and $\alpha$ is a continuously varying parameter.

The case with $\alpha=0$ is the usual BA model. For the negative values of $\alpha$, the largest value of the modulation factor $\ell^{\alpha}$ corresponds to the smallest value of $\ell$. Therefore, in the limit of $\alpha \rightarrow-\infty$, only the smallest value of $\ell$ corresponding to the nearest node will contribute with probability one. Similarly, for $\alpha>0$ large $\ell$ values will 


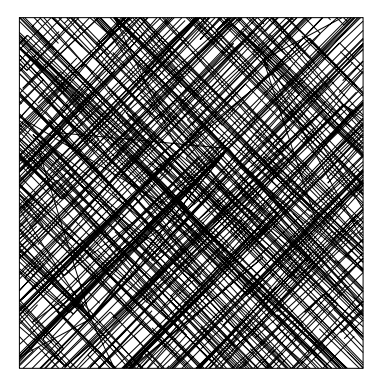

$\alpha=+\infty$

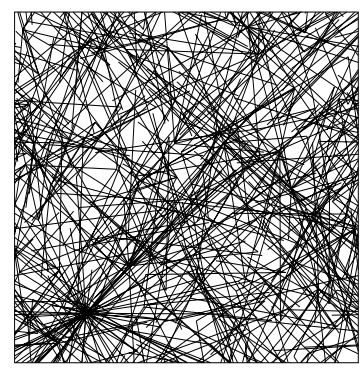

$\alpha=0$

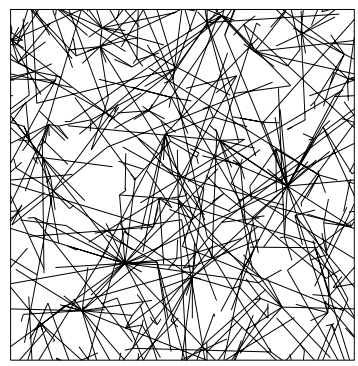

$\alpha=-2$

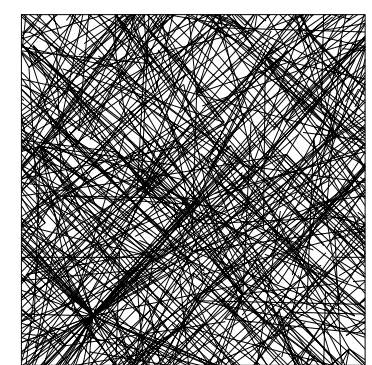

$\alpha=+5$

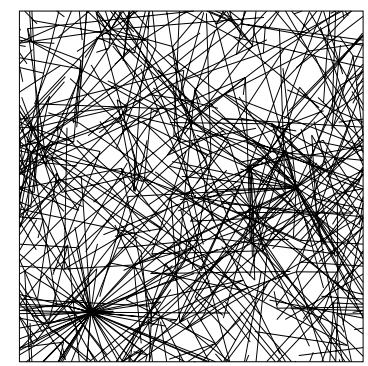

$\alpha=-1$

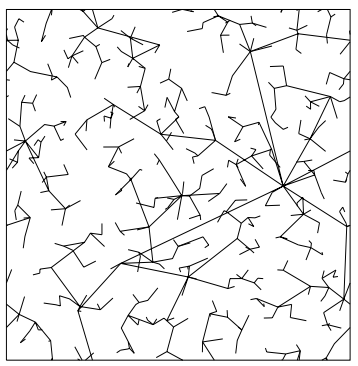

$\alpha=-\infty$
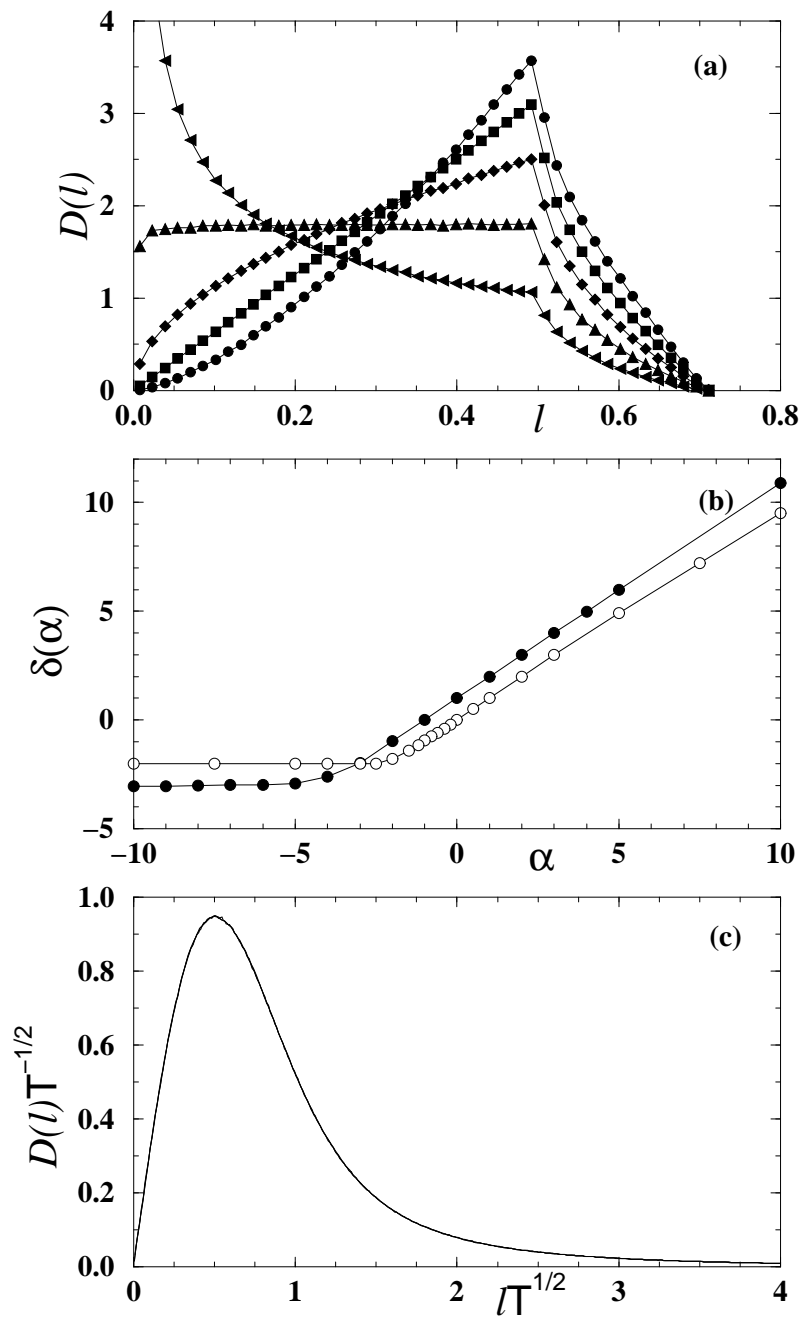

FIG. 1: Modulated scale free networks within an unit square for different values of the modulation parameter $\alpha$ for the same distribution of 512 nodes. For $\alpha=+\infty$ and $-\infty$ a newly introduced node is linked only to its farthest and nearest predecessors respectively, where as for $\alpha=0$ it is connected to one of the previous node according to the BA rule.

be more probable and the limit of $\alpha \rightarrow+\infty$ corresponds to only non-zero contribution from the furthest node.

We start with only one initial node corresponding to $m=m_{o}=1$ and connect a new node to only one of its previous nodes. Therefore, every node has only one outgoing link $\left(k_{\text {out }}=1\right)$ but can have a number of incoming links $\left(k_{i n}\right)$. The network thus formed has a tree structure, without any loops. Similar to BA model we expect that the main results of our model should be robust with respect to the value of $m_{o}$ used [B].

The link lengths of this network vary over a wide range. We define $D(\ell) d \ell$ as the probability that a randomly selected link has the length between $\ell$ and $\ell+d \ell$ and assume a power law distribution $D(\ell) \sim \ell^{\delta}$. Since the network with $\alpha=0$ has no length dependence and since the nodes are located in random positions in space with uniform
FIG. 2: (a) The probability density distribution $D(\ell)$ of the link lengths $\ell$ in $d=2$ for five different $\alpha$ values: $1 / 2$ (circle), 0 (square), $-1 / 2$ (diamond), -1 (triangle up), $-3 / 2$ (triangle left) for networks of $\mathcal{T}=2^{12}$ (b) $\delta(\alpha)$ varies linearly with $\alpha$ for $\alpha>-4$ in $d=2$ and for $\alpha>-2$ in $d=1$. The saturation values of $\delta$ are -3 and -2 for $d=2$ (filled circle) and $d=1$ (opaque circle) respectively. (c) Scaling of the probability density for $\alpha=-\infty$ and for $\mathcal{T}=2^{11}, 2^{13}$ and $2^{15}$ in $d=2$.

probabilities, $D(\ell)$ for $\alpha=0$ should depend only on the volume of the spherical shell between $\ell$ and $\ell+d \ell$ and $D(\ell) \sim \ell^{d-1}$ in the $d$-dimensional space. For $\alpha \neq 0$, this distribution is modified by the factor $\ell^{\alpha}$ of Eqn. 3 giving

$$
D(\ell) \sim \ell^{\alpha+d-1}
$$

which implies $\delta(\alpha)=\alpha+d-1$. Therefore at a particular value of $\alpha=\alpha_{c}=1-d, \delta(\alpha)=0$ and the distribution is uniform in any dimension. $D(\ell)$ grows with $\ell$ for $\alpha>$ $\alpha_{c}$ and decays with $\ell$ for $\alpha<\alpha_{c}$. Growing, uniform and decaying distributions are shown in Fig. 2(a) for different values of $\alpha$ which confirm $\alpha_{c}=-1$ in $d=2$. 

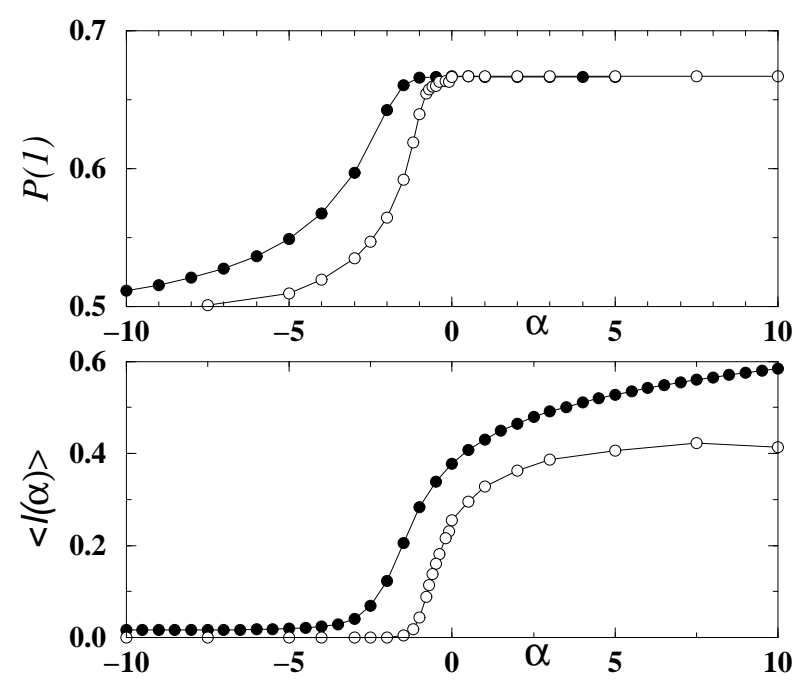

FIG. 3: For networks with $\mathcal{T}=2^{12}$, variations of (a) the probability $P(1)$ of a node of degree 1 (b) average length $\ell(\alpha)$ of a link with $\alpha$. Opaque circles for $d=1$ and the filled circles are for $d=2$.

Similar calculations in $d=1$ show uniform distribution for $\alpha_{c}=0$.

Since we distribute nodes within the unit square and link lengths are measured using the periodic boundary conditions along both the $x$ and $y$ directions, the distance between any two nodes can be at most $\ell_{o}=2^{-1 / 2}$. Consequently all orientations of links of lengths up to $1 / 2$ are equally likely. However links of lengths greater than $1 / 2$ have to be oriented more towards to the diagonal directions i.e., $y= \pm x$ lines to properly fit in and therefore their orientations are not equally likely. This anisotropic effect is observed in $d=2$ when $D(\ell)$ decays at a faster rate for $1 / 2<\ell<\ell_{o}$ since isotropy of the orientation of these links is lost and therefore they are less probable. In contrast, we do not see such a region in one dimension in agreement with the theoretical analysis.

However, $\delta(\alpha)$ saturates at a minimum value $\delta_{m}$ below a certain value of $\alpha$ which is calculated exactly in the limit of $\alpha \rightarrow-\infty$. Since, in this limit a new node is always connected to its closest neighbour, the probability that the $t+1$-th node has a link of length between $\ell$ and $\ell+d \ell$ is

$$
D_{t}(\ell) d \ell=a \ell^{d-1} t\left(1-b \ell^{d}\right)^{t-1} d \ell
$$

where $b \ell^{d}$ is the volume of a hypersphere in $d$ dimensions and $a=b d$. Therefore for a network evolved up to a time $\mathcal{T}$, the link length distribution of the whole network is

$$
D(\ell) d \ell=\Sigma_{t=1}^{\mathcal{T}} D_{t}(\ell) d \ell=a \ell^{d-1} d \ell \Sigma_{t=1}^{\mathcal{T}} t\left(1-b \ell^{d}\right)^{t-1} .
$$

In the limit $\mathcal{T} \rightarrow \infty$ this series converges for large $\ell$ to $D(\ell) \sim \ell^{-d-1}$ giving $\delta_{m}=-(d+1)$. However, for small $\ell$,

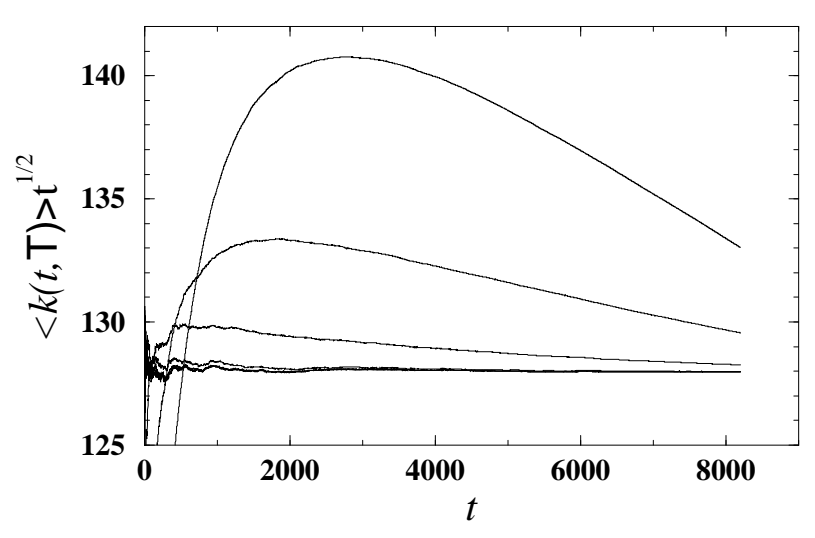

FIG. 4: Average degree of the node $\langle k(t, \mathcal{T})\rangle$ at time $t$ for a two dimensional network of duration $\mathcal{T}=2^{14}$ multiplied by $t^{1 / 2}$ is plotted for different $\alpha$. For $\alpha=3,2,1,-1$ plots are parallel to the $t$ axis for large $t$. The curves deviate for $\alpha=-3 / 2,-2$ and for -3 .

$D(\ell)=c_{1} \ell^{d-1}-c_{2} \ell^{2 d-1}$ ignoring higher order corrections where $c_{1} \sim O\left(t^{2}\right)$ and $c_{2} \sim O\left(t^{3}\right)$ for large $t$. This implies that $D(\ell)$ must have a maximum at $\ell \sim \mathcal{T}^{-1 / d}$ for all $d>1$ as verified below by the scaling analysis. For one dimension however no such maximum is expected and the power law decay starts right from the small values of $\ell$. Again since $\delta$ varies linearly with $\alpha$ as $\delta(\alpha)=\alpha+d-1$ in general, the minimum value $\delta_{m}$ is attained at $\alpha=-2 d$ and remains same for smaller values of $\alpha$.

Our numerical findings nicely support these results: we check that $\delta$ saturates nearly at $\delta_{m}=-3$ for $\alpha<-4$ for $d=2$ and $\delta_{m}=-2$ for $\alpha<-2$ in $d=1$ (Fig. 2(b)). We look at the distribution in more detail in $d=2$ for small values of $\ell$. On decreasing $\alpha$ from -1 a maximum appears at $\ell \approx 0.01$ (Fig. 2(c)) i.e., as $\ell$ increases from zero the distribution grows very rapidly as a power law, reaches a maximum and then decays. For a particular value of $\alpha$, $D(\ell)$ scales nicely with the duration $\mathcal{T}$ of growth as:

$$
D(\ell) \sim \mathcal{T}^{1 / 2} \mathcal{G}\left(\ell \mathcal{T}^{1 / 2}\right)
$$

In Fig. 2(c) we plot this collapsed data which fits very well to the following form of the scaling function:

$$
\mathcal{G}(x)=a^{\prime} x^{b^{\prime}} /\left(x^{2}+c^{\prime}\right)^{d^{\prime}} .
$$

Values of all the constants $a^{\prime}, b^{\prime}, c^{\prime}, d^{\prime}$ of this expression are dependent on $\alpha$. No such maximum in $D(\ell)$ at the small values of $\ell$ is observed in one dimension.

We studied the cumulative degree distribution $F(k)=$ $\int_{k}^{\infty} P(k) d k \sim k^{1-\gamma}$ and assume the following scaling behaviour:

$$
F(k, \mathcal{T}) \sim \mathcal{T}^{\eta} \mathcal{F}\left(k / \mathcal{T}^{\zeta}\right)
$$

where the scaling function $\mathcal{F}(x) \rightarrow x^{1-\gamma}$ when $x<<1$ and $\mathcal{F}(x) \rightarrow$ constant for $x>>1$. This implies that 
$\gamma=1+\eta / \zeta$. For example for $\alpha=-1$ at $d=2$ a good data collapse is obtained for $\eta=1$ and $\zeta=1 / 2$ giving $\gamma=3$ and the same result is obtained for all values of $\alpha>-1$. However for $\alpha<-1$ we see a stretched exponential variation $P(k) \sim \exp \left(-k^{\psi(\alpha)}\right)$ and $\psi(\alpha)$ increases to 1 i.e., to a pure exponential form at $\alpha=-\infty$. It therefore appears that the transition from the stretched exponential to the scale-free behaviour is perhaps taking place at the specific value of $\alpha_{c}=-1$ in $d=2$ and in general at $\alpha_{c}=1-d$ in the $d$ dimension. However a similar study in one dimension shows all indications that $\alpha_{c}$ is very likely to be around -0.5 , certainly greater than -1 but seems to be smaller than zero. Therefore we conjecture that $\alpha_{c}=1-d$ though our numerical analysis in one dimention does not fully confirm this prediction. At the opposite limit of $\alpha=\infty$ i.e. when each node is connected to its farthest neighbour, the degree distribution is found to be exponentially decaying and it appears that it happens only at $\alpha=\infty$ since even at $\alpha=40$ we found scaling of the distribution.

We note a few more important properties of this network near $\alpha_{c}$. The first moment of the degree distribution $\langle k\rangle$ is exactly 2 since the sum of the degrees of all nodes is $2 \mathcal{T}$ counting each link twice where as the number of nodes is $\mathcal{T}+1$. As the cut-off of the degree distribution varies as $\mathcal{T}^{\zeta}$ the $\langle k\rangle$ is defined as $\int_{1}^{\mathcal{T}^{\zeta}} k P(k) d k / \int_{1}^{\mathcal{T}^{\zeta}} P(k) d k$. Assuming $\gamma>2$ the mean is $(\gamma-1) /(\gamma-2)$ in the large $\mathcal{T}$ limit. This gives $\gamma=3$ correctly. Since each node has exactly one outgoing link, the mean number of incoming links averaged over all nodes must be equal to 1 . The fraction of nodes with nonzero incoming links should be 1- $P(1)$. In Fig. 3(a) we plot the variation of $P(1)$ with $\alpha$. This varies from 0.4777 at $\alpha=-\infty$ to very close to $2 / 3$ for $\alpha>\alpha_{c}$. Therefore the mean number of incoming links per node $\left\langle k_{i n}(\alpha)\right\rangle$ averaged over all nodes with $k_{i n} \neq 0$ is $\mathcal{T} /[(1-P(1)) \mathcal{T}]=3$ for $\alpha>\alpha_{c}$. This is checked numerically. We also keep track of the variation of average link length $\langle\ell(\alpha)\rangle$ as the characteristic distance of this system which is shown in Fig. 3(b). Since for $\alpha<\alpha_{c}$ smaller links are more probable, $\langle\ell(\alpha)\rangle$ is very small and approaches zero as $\mathcal{T}^{-1 / 2}$. For $\alpha>\alpha_{c},\langle\ell(\alpha)\rangle$ grows with $\alpha$. Finally the fraction of anisotropic links $f(\ell>1 / 2)$ also grows from zero at $\alpha=\alpha_{c}$.

The average degree of a node which is introduced at the time $t$ to a network which has grown up to a time $\mathcal{T}$ is denoted by $\langle k(t, \mathcal{T})\rangle$ and follows a power law variation with $t$ at large $t$ values as: $\langle k(t, \mathcal{T})\rangle \sim t^{\beta}$. For BA network, $\beta=1 / 2$ is obtained [11] and it is connected to the degree distribution exponent $\gamma$ by the relation: $\beta(\gamma-1)=1$. We have calculated $\langle k(t, \mathcal{T})\rangle$ in two dimensions for different values of $\alpha$ and in Fig. 4 we plot $\langle k(t, \mathcal{T})\rangle t^{1 / 2}$ vs. $t$ on a linear scale. We observe that for all dalues of $\alpha>-1$ the plot is horizontal implving that $\beta=1 / 2$ for this range of $\alpha$ values.

To summarize, we studied a growing random network where the attachment probability $\pi_{i}$ to a previous node $i$ depends jointly on the degree of the node $k_{i}$ as well as on the $\alpha$-th power of the link-length $\ell$ as $\pi_{i} \sim k_{i} \ell^{\alpha}$. By tuning $\alpha$ we find that for $\alpha<\alpha_{c}$, the degree distribution of the resulting network is stretched exponential whereas for $\alpha>\alpha_{c}$ the network is scale-free. We also observe that the link length distribution follows a power law: $D(\ell) \sim \ell^{\delta}$ for the whole range of the parameter $\alpha$ in contrast to the Waxman's exponential distribution [12. The exponent $\delta$ grows linearly with $\alpha$ for $\alpha \geq-2 d$ and saturates at $-(d+1)$ for $\alpha<-2 d$. Our interesting observation is when $\alpha=-d$ the network has the property of real internet network where the link length distribution varies inversely with the link length.

We thank I. Bose, A. Chatterjee, S. Dasgupta and P. A. Sreeram for many useful discussions on network related problems. PS acknowledged financial support from DST grant SP/S2-M11/99.

Electronic Address: manna@boson.bose.res.in parongama@vsnl.net

[1] S. Lawrence and C. L. Giles, Science, 280, 98 (1998); Nature, 400, 107 (1999), R. Albert, H. Jeong and A.-L. Barabási, Nature, 401, 130 (1999).

[2] M. Faloutsos, P. Faloutsos and C. Faloutsos, Proc. ACM SIGCOMM, Comput. Commun. Rev., 29, 251 (1999).

[3] J. J. Hopfield and A. V. M. Herz, Proc. Natl. Acad. Sci. USA, 92, 6655 (1995).

[4] M. E. J. Newman. Proc. Nat. Acad. Sci. USA, 98, 404 (2001); arXiv:cond-mat/0011155.

[5] P. Erdös and A. Rényi, Publ. Math. Debrecen, 6, 290 (1959).

[6] D. J. Watts and S. H. Strogatz, Nature, 393, 440 1998; D. J. Watts, Small Worlds: The Dynamics of Networks Between order and Randomness, (Princeton 1999).

[7] A.-L. Barabási and R. Albert, Science, 286, 509 (1999).

[8] R. Albert and A.-L. Barabási, Rev. Mod. Phys. 74, 47 (2002).

[9] S. Jespersen and A. Blumen, Phys. Rev. E 62, 6270 (2000); J. Kleinberg, Nature 406, 845 (2000); P. Sen and B. K. Chakrabarti, J. Phys. A 34, 7749 (2001); P. Sen, K. Banerji and T. Biswas, Phys. Rev. E. (in press), arXiv:cond-mat/0206570.

[10] J. Jost and M. P. Joy, arXiv:cond-mat/0202343; S. H. Yook, H. Jeong, A.-L. Barabási and Y. Tu, Phys. Rev. Lett. 86, 5835 (2001).

[11] S. N. Dorogovtsev and J. F. F. Mendes, Phys. Rev. E., 62, 1842 (2000); 63, 056125 (2001).

[12] B. Waxman, IEEE J. Selec. Areas Commun., SAC, 6, 1617 (1988).

[13] S.-H. Yook, H. Jeong and A.-L. Barabási, arXiv:condmat/0107417. 